\title{
Improved Reflectarray Phase-Only Synthesis Using the Generalized Intersection Approach with Dielectric Frame and First Principle of Equivalence
}

\author{
Daniel R. Prado, Manuel Arrebola, Marcos R. Pino, and Fernando Las-Heras \\ Department of Electrical Engineering, University of Oviedo, Edificio Polivalente, Módulo 8, 33203 Gijón, Spain \\ Correspondence should be addressed to Manuel Arrebola; arrebola@tsc.uniovi.es
}

Received 27 February 2017; Revised 10 April 2017; Accepted 30 April 2017; Published 23 May 2017

Academic Editor: Ahmed Toaha Mobashsher

Copyright @ 2017 Daniel R. Prado et al. This is an open access article distributed under the Creative Commons Attribution License, which permits unrestricted use, distribution, and reproduction in any medium, provided the original work is properly cited.

\begin{abstract}
An improved reflectarray Phase-Only Synthesis technique which employs the generalized Intersection Approach (IA) algorithm is fully described. It is formulated with the First Principle of Equivalence and takes into account a dielectric frame which is usually present to screw the reflectarray breadboard to the supporting structure. The effects of the First Principle of Equivalence versus the Second Principle in the computation of the radiation patterns, as well as the dielectric frame, are assessed and taken into account in an efficient implementation of the generalized IA in order to obtain more accurate results. Different strategies to speed up the synthesis process are presented and to improve convergence. The technique is demonstrated through two examples for space and terrestrial applications: an isoflux pattern for global Earth coverage from a satellite and a Local Multipoint Distribution Service pattern for central stations of cellular systems, both with a working frequency of $25.5 \mathrm{GHz}$. In addition, experimental results validate the approach described in this work with a prototype with an isoflux pattern working at $30 \mathrm{GHz}$.
\end{abstract}

\section{Introduction}

Design of reflectarray antennas has been a challenge for the past three decades. In particular, special efforts have been made regarding the synthesis of shaped-beam reflectarrays for different applications [1]. In this regard, the most common technique for the synthesis of reflectarray antennas (as well as phased-arrays) is the Phase-Only Synthesis (POS), which can be implemented by several algorithms, such as Intersection Approach [2, 3], Levenberg-Marquardt [4], steepest descent [5], conjugate gradient [6], genetic algorithm [7], or particle swarms optimization [8], among others. Despite the availability of several algorithms for the POS of reflectarrays, most of them present limitations regarding memory usage, convergence, or speed [4]. On this subject, the Intersection Approach (IA) [3] offers an extremely efficient algorithm since the most time-consuming operation is the Fast Fourier Transform (FFT) that is employed to compute the radiation pattern and to recover the tangential fields on the reflectarray surface. Furthermore, this algorithm has been used with success in the design of reflectarray antennas with very tight requirements for space applications [9-11]. However, this algorithm suffers from the problem of traps or local minima [12], although some strategies have been developed to minimize this issue [3]. Convergence of the IA can be improved by employing the generalized IA [12-14] working with the squared field amplitude (or equivalently, with the directivity or gain), although at the cost of greatly reducing its computational efficiency, since in that case a general minimization algorithm must be employed.

On the other hand, reflectarrays are classified as planar apertures and thus their radiation pattern can be computed by using the First, Second, or Third Principles of Equivalence. Most commonly, the Second Principle is used [1] since it only requires knowledge of the tangential electric field. Nevertheless, it has been recently proven that the use of the First Principle of Equivalence provides more accuracy in the prediction of the far field, especially in the crosspolar pattern [15]. This principle requires, in addition to the tangential electric field, knowledge about the magnetic tangential field. However, POS algorithms are usually implemented with the Second Principle [1]. Furthermore, reflectarray antennas 
usually have a dielectric frame to screw the breadboard to the supporting structure, and it is also backed by a ground plane. The dielectric frame may affect the copolar pattern and thus should be conveniently taken into account both in the analysis and synthesis.

In this paper, we propose an improved and practical reflectarray Phase-Only Synthesis based on the generalized Intersection Approach. The novelty of the approach is the development of the POS formulation for the First Principle of Equivalence as well as considering the dielectric frame in the synthesis process. The effects of both are assessed in the copolar pattern, demonstrating the need of including both influences in the antenna optimization. In addition, and in order to improve the computational efficiency of the generalized IA, some strategies to speed up computations are presented and discussed. The technique is demonstrated with two examples of shaped-beam reflectarrays, one for space applications with an isoflux pattern for global Earth coverage from a geostationary satellite and another for central stations of cellular systems with a Local Multipoint Distribution Service (LMDS) pattern. Finally, an experimental validation is provided with a reflectarray prototype with an isoflux pattern, in which the effect of the First Principle and dielectric frame are patent in the coverage area.

The paper is organized as follows. Section 2 includes the reflectarray analysis, including that of the dielectric frame. Section 3 describes the generalized IA for reflectarray POS, particularizing the analysis from the previous section. It also introduces two strategies to improve convergence and computing time. Section 4 includes the results assessing the effects of the First Principle of Equivalence and dielectric frame on the copolar pattern and the synthesis of two shapedbeam reflectarrays to demonstrate the technique as well as the experimental validation. Finally, Section 5 presents the conclusions.

\section{Reflectarray Analysis}

2.1. Tangential Field on the Aperture. The sketch of a singleoffset reflectarray configuration is shown in Figure 1. The reflectarray is illuminated by a primary feed (usually a horn antenna) generating an incident electric field on its surface. The tangential reflected field on the reflectarray surface at each reflectarray element $(m, n)$ can be expressed as [14]

$$
\vec{E}_{\mathrm{ref}}^{X / Y}\left(x_{m}, y_{n}\right)=\mathbf{R}^{m n} \cdot \vec{E}_{\mathrm{inc}}^{X / Y}\left(x_{m}, y_{n}\right),
$$

where $\mathbf{R}^{m n}$ is the reflection coefficient matrix, $\vec{E}_{\text {inc }}^{X / Y}\left(x_{m}, y_{n}\right)$ the incident field impinging from the feed, the superscripts indicate the polarization of the field, and $\left(x_{m}, y_{n}\right)$ are the coordinates of the $(m, n)$ th element. The components of matrix $\mathbf{R}^{m n}$ are complex numbers which fully characterize the behavior of the unit cell. This matrix takes the form

$$
\mathbf{R}^{m n}=\left(\begin{array}{cc}
\rho_{x x}^{m n} & \rho_{x y}^{m n} \\
\rho_{y x}^{m n} & \rho_{y y}^{m n}
\end{array}\right),
$$

where $\rho_{x x}^{m n}$ and $\rho_{y y}^{m n}$ are known as direct coefficients, while $\rho_{x y}^{m n}$ and $\rho_{y x}^{m n}$ are the cross-coefficients. They are computed with a full-wave analysis tool assuming local periodicity [1].

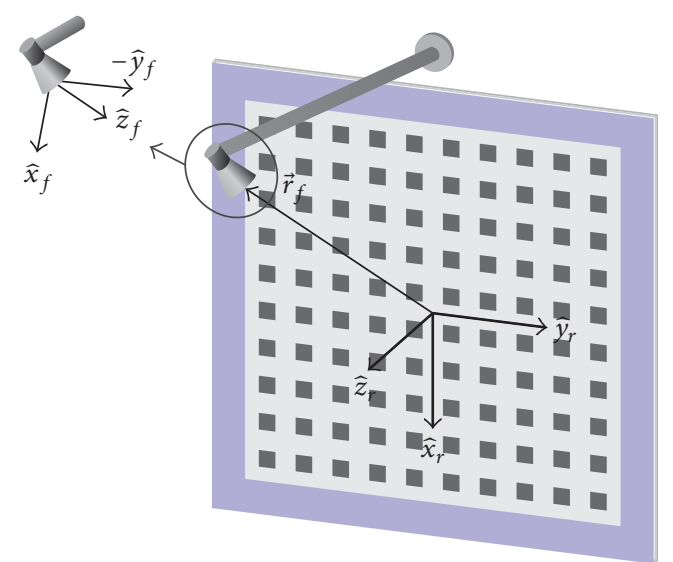

FIGURE 1: Sketch of the reflectarray single-offset geometry under study, with the dielectric frame in light blue color. It holds $\widehat{y}_{r}=-\hat{y}_{f}$.

The First Principle of Equivalence requires the computation of the reflected tangential magnetic field. It can be computed at each reflectarray element assuming a locally incident plane wave coming from the feed, using the electric reflected field of (1) following [14].

2.2. Computation of the Radiation Patterns. Once the tangential reflected field has been obtained, the radiation patterns are computed. Reflectarrays are classified as planar apertures and the far fields can be efficiently calculated by using the FFT algorithm. Following [1], the electric and magnetic spectrum functions can be expressed as Inverse Discrete Fourier Transforms of the tangential reflected fields as follows:

$$
\begin{aligned}
& P_{x / y}^{X / Y}(u, v)=N \cdot K \cdot \operatorname{IDFT2}\left[E_{\mathrm{ref}, x / y}^{X / Y}\left(x_{m}, y_{n}\right)\right], \\
& Q_{x / y}^{X / Y}(u, v)=N \cdot K \cdot \operatorname{IDFT2}\left[H_{\mathrm{ref}, x / y}^{X / Y}\left(x_{m}, y_{n}\right)\right],
\end{aligned}
$$

with $N$ being the total number of reflectarray elements and $K$ being a constant which accounts for the active element pattern. With the compact notation used in (3), there are four spectrum functions per polarization ( $x$ and $y$ components for $P$ and $Q$ ), having a total of eight spectrum functions for dualpolarized reflectarrays.

Then, the radiation patterns are obtained in spherical coordinates by using the First Principle of Equivalence [16]. The copolar and crosspolar components of the far field are obtained by applying Ludwig's third definition of crosspolarization [17] for both linear polarizations. Finally, the gain can be estimated by computing the total power radiated by the feed, as in [1].

2.3. Analysis of the Dielectric Frame. When manufacturing a reflectarray breadboard, it is very common that a dielectric frame is included. This frame is also backed by a ground plane, but has no metallization, and is employed to screw the breadboard to the supporting structure. Even though the field level at the edge of the reflectarray is quite low, usually below the optimum level of $-12 \mathrm{~dB}$ which optimizes illumination efficiency [18] in order to minimize diffraction effects [1], 


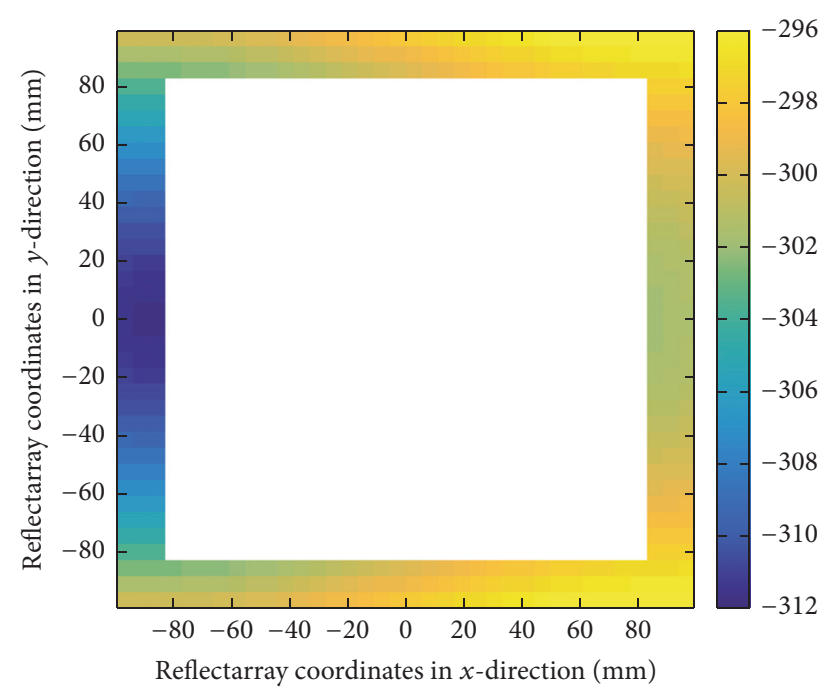

FIGURE 2: Phase shift in degrees introduced by a dielectric frame comprised of three extra elements at the edge of the reflectarray for $X$ polarization. The frame is modeled as extra rows and columns added at the reflectarray edge and can alter the radiation pattern if it is not taken into account in the analysis and synthesis.

modeling the frame is important to correctly characterize the radiated fields. In this regard, when including the dielectric frame in the analysis and synthesis, the substrate must be selected beforehand, since its characteristics will determine its response to the incident field coming from the feed. The dielectric frame can be analyzed very efficiently since there is no metallization and the analysis is greatly simplified [19]. The dielectric frame will present some losses due to the dielectric tangent loss, although they will be very low. More important is the phase shift introduced by the frame at each position, which will vary with the angle of incidence from the feed and the feed position, and can modify the copolar and crosspolar patterns when compared with the analysis with no dielectric frame. Also, the phase shift is different for each polarization [1], changing not only the distribution of the phase on the dielectric frame but also the total range.

To simplify the analysis, the dielectric frame is specified as a number of elements added as extra rows and columns to the edge of the reflectarray. Usually, a frame comprised of one, two, or three extra elements at each side is enough [15], but there are cases in which the frame is made up of an elevated number of extra rows and columns, such as in [20] where the frame is comprised up to 11 extra elements at each side of the reflectarray, which should be conveniently taken into account in the analysis and synthesis.

An example is shown in Figure 2, which was generated adding six extra rows (three at the upper edge and three at the lower edge) and six extra columns (three at the left edge and three at the right edge). It corresponds to two stacked layers of CuClad 233, each one with thickness $0.787 \mathrm{~mm}, \epsilon_{r}=2.33$, and $\tan \delta=0.0013$. The feed is placed at $(-94,0,214) \mathrm{mm}$ with regard to the center of the reflectarray and the real angle of incidence is taken into account in the analysis. The frame shown in Figure 2 and the above-mentioned substrate will be employed in later sections for the synthesis of different shaped beams.

\section{Generalized Intersection Approach for POS}

The chosen algorithm is the generalized Intersection Approach (IA) [14] for Phase-Only Synthesis. It is an iterative algorithm which performs two operations at each iteration $i$ on the radiated field $\vec{E}_{i}$ :

$$
\vec{E}_{i+1}=\mathscr{B}\left[\mathscr{F}\left(\vec{E}_{i}\right)\right],
$$

where $\mathscr{F}$ is known as the forward projector, which projects the radiated field by the antenna onto the set of fields which comply with the specifications; and $\mathscr{B}$ is the backward projector, which projects the field which complies with the specifications onto the set of fields which can be radiated by the antenna. The goal is to find a field which belongs simultaneously to both sets, or if that is not possible because the intersection between both sets is void, to find a field with whose distance to the set of fields which comply with the requirements is minimum.

A fast implementation which employs the FFT in both projectors is presented in $[3,21]$. However, this implementation suffers from the problem of traps, or local minima, which penalizes its convergence. Furthermore, it can only be implemented for POS with the Second Principle of Equivalence, as it will be shown later. These two limitations can be overcome by employing the generalized IA (which allows to employ the First Principle) and working with the squared field amplitude instead of the field amplitude as in $[3,21]$ (which minimizes the problem of traps and improves convergence). However, the computational efficiency of the FFT is lost in the backward projector, and thus new strategies to speed up computations will be introduced to compensate for this fact.

\subsection{Forward Projection}

3.1.1. Computation of the Far Fields. The first step in the implementation of the generalized IA is the definition of the forward projector, whose first operation is the computation of the radiation patterns, for both linear polarizations. In order to carry a POS, some simplifications in the reflectarray analysis are necessary. First, the reflectarray unit cell is modeled as an ideal phase shifter [4], where there are no losses (i.e., $\left|\rho_{x x}^{m n}\right|=\left|\rho_{y y}^{m n}\right|=1$ ) and no element crosspolarization (i.e., $\left.\rho_{x y}^{m n}=\rho_{y x}^{m n}=0\right)$. With these two approximations, the $\mathbf{R}^{m n}$ matrix is simplified to

$$
\mathbf{R}^{m n}=\left(\begin{array}{cc}
\exp \left(j \phi_{x x}^{m n}\right) & 0 \\
0 & \exp \left(j \phi_{y y}^{m n}\right)
\end{array}\right),
$$

where $\phi^{m n}$ is the phase of the corresponding reflection coefficient. Using (5) in (1), the reflected field of each polarization still depends on the phases of both direct coefficients, $\phi_{x x}^{m n}$ and $\phi_{y y}^{m n}$. However, it is interesting to have the synthesis process independent for both polarizations [1]. Hence, the 
cross component of the incident wave is assumed to be zero ( $\hat{y}$ component in $X$ polarization and $\hat{x}$ component in $Y$ polarization). This simplification is reasonable since it holds that $\left|E_{\mathrm{inc}, x}^{X}\right| \gg\left|E_{\mathrm{inc}, y}^{X}\right|$ and $\left|E_{\mathrm{inc}, y}^{Y}\right| \gg\left|E_{\mathrm{inc}, x}^{Y}\right|$. Thus, the final tangential electric field at the aperture is

$$
\begin{aligned}
& E_{\mathrm{ref}, x}^{X}\left(x_{m}, y_{n}\right)=\exp \left(j \phi_{x x}^{m n}\right) E_{\mathrm{inc}, x}^{X}\left(x_{m}, y_{n}\right), \\
& E_{\mathrm{ref}, y}^{Y}\left(x_{m}, y_{n}\right)=\exp \left(j \phi_{y y}^{m n}\right) E_{\text {inc, } y}^{Y}\left(x_{m}, y_{n}\right) .
\end{aligned}
$$

The simplified tangential magnetic field is obtained from (6). The $\widehat{z}$ component of the electric field is obtained from the plane wave relation $\vec{k}_{\text {ref }} \cdot \vec{E}_{\text {ref }}=0$ [14]. For $X$ polarization, this leads to

$$
E_{\mathrm{ref}, z}^{X}=-\frac{k_{\mathrm{ref}, x} E_{\mathrm{ref}, x}^{X}}{k_{\mathrm{ref}, z}} .
$$

The tangential components of the reflected magnetic field are then obtained solving the cross-product of $\vec{k}_{\text {ref }}$ and $\vec{E}_{\text {ref }}[14]$, finally having

$$
\begin{aligned}
& H_{\mathrm{ref}, x}^{X}=\frac{k_{\mathrm{ref}, y} E_{\mathrm{ref}, z}^{X}}{\omega \mu_{0}}, \\
& H_{\mathrm{ref}, y}^{X}=\frac{-k_{\mathrm{ref}, x} E_{\mathrm{ref}, z}^{X}+k_{\mathrm{ref}, z} E_{\mathrm{ref}, x}^{X} .}{\omega \mu_{0}} .
\end{aligned}
$$

For $Y$ polarization, the process is analogous.

With these simplifications in the tangential fields for POS, $X$ polarization requires the computation of $P_{x}^{X}, Q_{x}^{X}$, and $Q_{y}^{X}$, while $Y$ polarization requires $P_{y}^{Y}, Q_{x}^{Y}$, and $Q_{y}^{Y}$. However, all spectrum functions depend only on $\phi_{x x}$ or $\phi_{y y}$ for $X$ polarization and $Y$ polarization, respectively, thus making each polarization independent of each other, as it happened with the formulation for the Second Principle of Equivalence [4]. This fact facilitates the design process of dual-polarized reflectarray antennas, since the copolar far field can be controlled independently for each polarization with the phase of one reflection coefficient, namely, $\phi_{x x}$ for $X$ polarization and $\phi_{y y}$ for $Y$ polarization.

Finally, since the POS the spectrum functions $P_{y}^{X}$ and $P_{x}^{Y}$ are zero, the simplified far fields using the First Principle of Equivalence for $X$ polarization are

$$
\begin{aligned}
& E_{\theta}^{X}=A\left[P_{x}^{X} \cos \varphi-\eta \cos \theta\left(Q_{x}^{X} \sin \varphi-Q_{y}^{X} \cos \varphi\right)\right], \\
& E_{\varphi}^{X}=-A\left[P_{x}^{X} \sin \varphi \cos \theta+\eta\left(Q_{x}^{X} \cos \varphi+Q_{y}^{X} \sin \varphi\right)\right],
\end{aligned}
$$

while, for $Y$ polarization, they are

$$
\begin{aligned}
& E_{\theta}^{Y}=A\left[P_{y}^{Y} \sin \varphi-\eta \cos \theta\left(Q_{x}^{Y} \sin \varphi-Q_{y}^{Y} \cos \varphi\right)\right], \\
& E_{\varphi}^{Y}=A\left[P_{y}^{Y} \cos \varphi \cos \theta-\eta\left(Q_{x}^{Y} \cos \varphi+Q_{y}^{Y} \sin \varphi\right)\right],
\end{aligned}
$$

where $\eta=\mu_{0} c_{0}$ is the vacuum impedance and

$$
A=\frac{j k_{0} e^{-j k_{0} r}}{4 \pi r} \text {. }
$$

On the other hand, using the Second Principle of Equivalence, the simplified far fields for $X$ polarization take the following form:

$$
\begin{aligned}
& E_{\theta}^{X}=2 A \cos \varphi P_{x}^{X}, \\
& E_{\varphi}^{X}=-2 A \cos \theta \sin \varphi P_{x}^{X},
\end{aligned}
$$

while, for $Y$ polarization, the radiated fields are given by the following:

$$
\begin{aligned}
& E_{\theta}^{Y}=2 A \sin \varphi P_{y}^{Y}, \\
& E_{\varphi}^{Y}=2 A \cos \theta \cos \varphi P_{y}^{Y},
\end{aligned}
$$

where $A$ is the same as in (11). From the far field in spherical coordinates, the copolar component for both polarizations is obtained.

3.1.2. Trimming the Far Field. Once the copolar far field has been obtained, the squared field amplitude or gain is computed. The last step of the forward projection is to trim it according to the given specification templates. The result of the forward projection is a far field which complies with the specifications, after being trimmed by the forward projector $[2,3,14,21]$, but that in general cannot be radiated by the antenna.

3.2. Backward Projection. The forward projection is extremely efficient since its most time-consuming operation is the FFT. Other implementations of the IA also employ the FFT in the backward projector to recover the tangential field $[2,3,21]$. However, that is only possible when the forward projector works with the field amplitude and when using the Second Principle of Equivalence. In that case, each copolar field only depends on one spectrum function and thus the spectrum functions can be recovered from them. In addition, the inverse operation of (3) can only be done working with the field amplitude.

In this paper, the generalized IA employs the First Principle and works with the squared field amplitude. Thus, the backward projection is redefined as the minimization of a distance:

$$
\vec{E}_{i+1}=\min \operatorname{dist}\left(\vec{E}_{i}, \mathscr{F}\left(\vec{E}_{i}\right)\right) \text {. }
$$

In the present case, the efficient implementation of the Levenberg-Marquardt Algorithm (LMA) of [4] has been chosen to perform the minimization in (14). The optimization variables will be the phases of the reflection coefficients, $\phi_{x x}$ for $X$ polarization and $\phi_{y y}$ for $Y$ polarization. The two polarizations can be synthesized independently.

3.3. Speeding Up the Algorithm. The generalized IA improves the convergence properties of the IA by working with the squared field amplitude. However, this is done at the cost of losing the characteristic computational efficiency since now a general minimization algorithm is needed in the backward projector. It is thus interesting to speed up the 
algorithm as much as possible because it allows saving time for a given problem size or tackling larger problems in the same amount of time. In this regard, the LMA used here [4] already addresses some concerns in the implementation of the algorithm, employing parallelization strategies in different building blocks and others.

Here, two new strategies to speed up the algorithm are discussed, the reduction of the number of optimizing variables and the resolution of the far fields. Both strategies reduce the size of the Jacobian matrix $(J)$, which is involved in the two most time-consuming operations of the LMA, the evaluation of $J$ and the matrix multiplication $J^{T} J$. First, the number of columns of $J$ is exactly the number of optimizing variables. And second, the number of rows of $J$ is the number of points in which the far field is discretized. By reducing the size of $J$, the LMA is sped up and uses less memory.

3.3.1. Reduction of the Number of Optimizing Variables. The first approach to reducing the size of the Jacobian is to reduce the number of optimizing variables. In addition to accelerating the algorithm, by doing the synthesis in several steps, optimizing a few variables at the beginning and increasing their number in following steps as the copolar pattern is shaped, convergence is improved [12, 13], since the number of local minima is reduced in the first steps of the synthesis. Finally, if the desired shaped pattern is symmetric, only half of the variables need to be optimized, further reducing computing time and memory.

3.3.2. Far Field Resolution. Reducing the far field resolution will also accelerate the evaluation of the Jacobian and the matrix multiplication. Since the far field is computed using the FFT algorithm, a grid with $2^{n} \times 2^{n}$ points is employed. Typical values for representation are $n=9,10[3,21]$ or even larger if the antenna has very high directivity. However, for small or medium-sized reflectarrays, the synthesis can be carried out for $n=7,8$, which substantially reduces computing time. In this regard, the synthesis can be performed in a UV grid with reduced resolution, and the representation of the results can be done with larger values of $n$.

Figure 3 shows a time study of the previous two strategies to reduce computing times. As it can be seen, the most important factor is the resolution of the far field, since the number of points in the UV grid decreases exponentially when $n$ diminishes. Reducing the number of optimizing variables has more impact in absolute terms for larger values of $n$. In addition, reducing the number of variables not only reduces computing time but also improves convergence of the overall synthesis process $[12,13]$. In any case, since any synthesis process may take tens or even hundreds of iterations, a modest improvement per iteration may have a significant impact in the whole procedure.

Finally, tests were carried out in an Intel Core i3-2100 with $4 \mathrm{CPU}$ (two physical and two virtual) at $3.1 \mathrm{GHz}$, from which data of Figure 3 was obtained. In comparison, each iteration in [21] took less than $0.5 \mathrm{~s}$ for a UV grid with $n=9$. This time is independent of the number of reflectarray elements and is slightly larger than the time which could be obtained with the

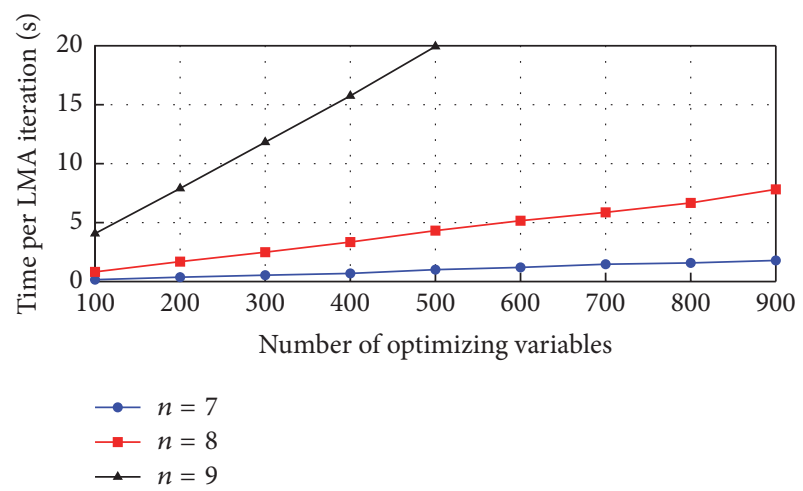

FIgURE 3: Computing time per LMA iteration depending on the number of optimizing variables and far field resolution for UV grids with $2^{n} \times 2^{n}$ points.

algorithm described in [3], since four more FFT are employed per iteration in [21]. Here, for $n=7$ and 900 optimizing variables, the mean time per iteration was $1.79 \mathrm{~s}$ and, for $n=8$, was $7.82 \mathrm{~s}$. However, the improved convergence properties of the generalized IA guarantee better results in less iterations $[13,22]$.

\section{Results}

4.1. Antenna Specifications. The considered reflectarray is rectangular and comprised of 900 elements in a rectangular grid of $30 \times 30$. The working frequency is $25.5 \mathrm{GHz}$ and the periodicity is $5.84 \mathrm{~mm} \times 5.84 \mathrm{~mm}$, which is approximately half a wavelength. The feed phase center is placed at $\vec{r}_{f}=$ $(-94,0,214) \mathrm{mm}$ with regard to the reflectarray center (see Figure 1) and is modeled as a $\cos ^{q} \theta$ function with $q=37$. For the dielectric frame analysis, two stacked layers of CuClad 233 , each one with thickness $0.787 \mathrm{~mm}, \epsilon_{r}=2.33$, and $\tan \delta=$ 0.0013 , are employed. In addition, the dielectric frame is comprised of two elements extra elements added at each side, as shown in Figure 2.

For the antenna synthesis, two different shaped beams are considered: first, an isoflux pattern [23] for global Earth coverage from a satellite in geostationary orbit with a tilt of $5.4^{\circ}$; second, a Local Multipoint Distribution Service pattern for base stations, with a squared cosecant pattern in elevation and sectorial beam in azimuth [24]. All syntheses were carried out with a far field resolution with $n=7$ to speed up computations, as well as using an increasing number of optimizing variables in successive steps. Furthermore, since the shape of the far fields is symmetric, it is also enforced the symmetry in the reflection coefficient phases, having to optimize only half the variables at each step. Once the synthesis is complete, the resulting far fields are computed with an increased resolution using $n=9$ to plot the results in the UV grid defined by $u=\sin \theta \cos \varphi, v=\sin \theta \sin \varphi$, where $(\theta, \varphi)$ are the usual far field spherical coordinates.

4.2. First versus Second Principle of Equivalence. In order to evaluate the differences of the copolar far fields computed by the First and Second Principles of Equivalence, syntheses 


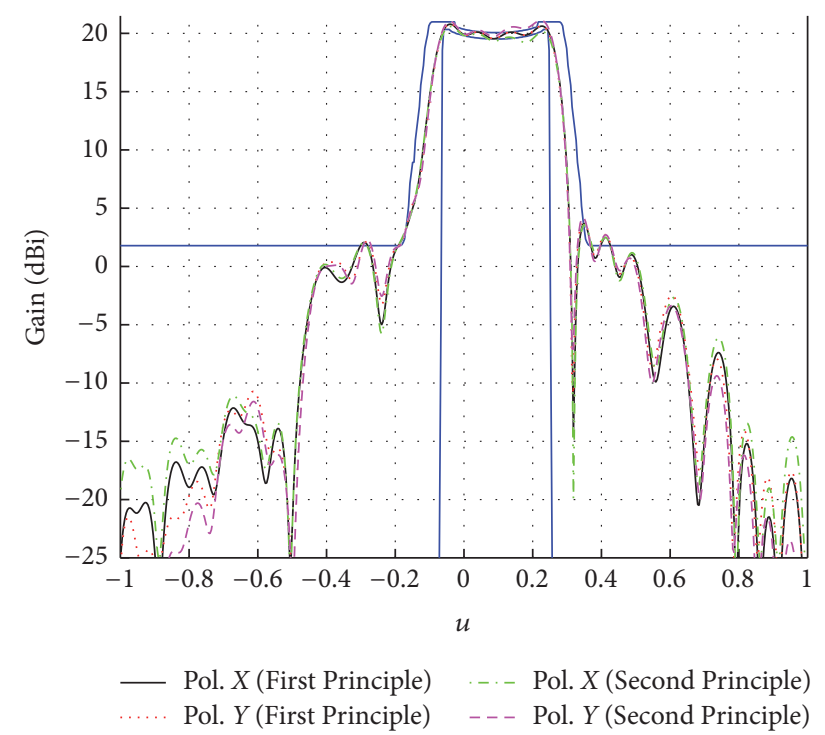

(a)

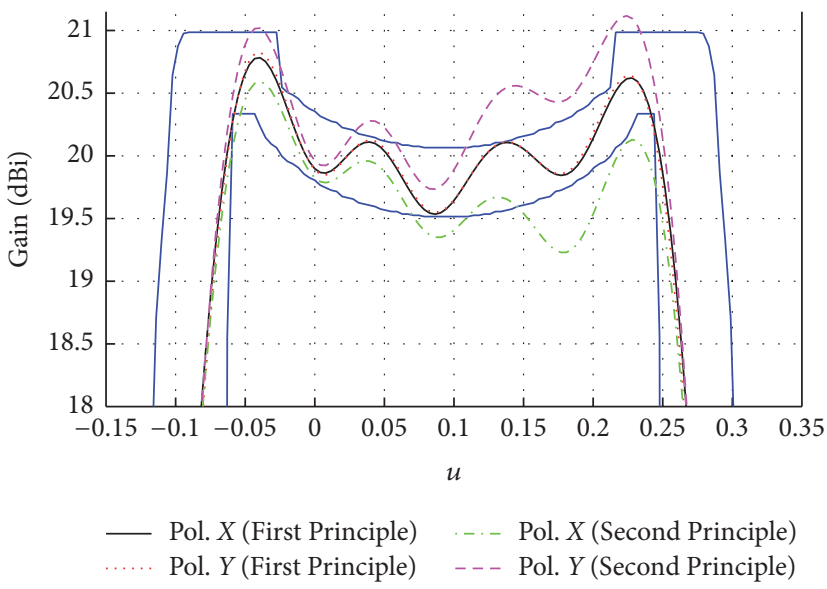

(b)

Figure 4: Differences between the First and the Second Principles of Equivalence for both linear polarizations in an isoflux pattern synthesized using the POS formulation of the First Principle. (a) Main cut in $u$. (b) Zoom on the coverage area.

of an isoflux and LMDS patterns were carried out using the POS formulation for the First Principle. Then, the resulting reflectarrays were simulated using both principles. Figure 4 shows the results for the isoflux pattern for the asymmetric cut. As it can be seen, both polarizations comply with the specifications when computed with the First Principle, since it was the one used in the synthesis. However, when they are computed using the POS formulation of the Second Principle of Equivalence, there are some discrepancies, and they are different depending on the polarization. If the synthesis was carried out using the Second Principle of Equivalence, the results shown in Figure 4 would be the opposite: the patterns computed with the Second Principle would comply with the requirements, while the patterns computed with the First Principle would not comply with the requirements. In addition, there are also discrepancies in the side lobes, which become more important for large angles far from broadside.

Figure 5 shows the results for the elevation cut for the LMDS pattern. This time there is a small discrepancy in the prediction of the gain using the Second Principle with regard to the First Principle, similar to what happens in Figure 4. In this case, the Second Principle predicts a gain $0.3 \mathrm{~dB}$ higher in $Y$ polarization and $0.3 \mathrm{~dB}$ lower in $X$ polarization, with respect to the corresponding polarizations with the First Principle. Also, the coverage area is seriously affected in wide angles. The effects in the azimuth cut are the same.

In light of these results, it is recommended to perform the POS using the formulation of the First Principle of Equivalence, since it provides more accurate results [15] since now the knowledge of the magnetic field is employed in the computation of the far field, and the simplification of the Second Principle is avoided.

4.3. Effect of the Dielectric Frame. To assess the effect of the dielectric frame, the isoflux and the LMDS patterns were

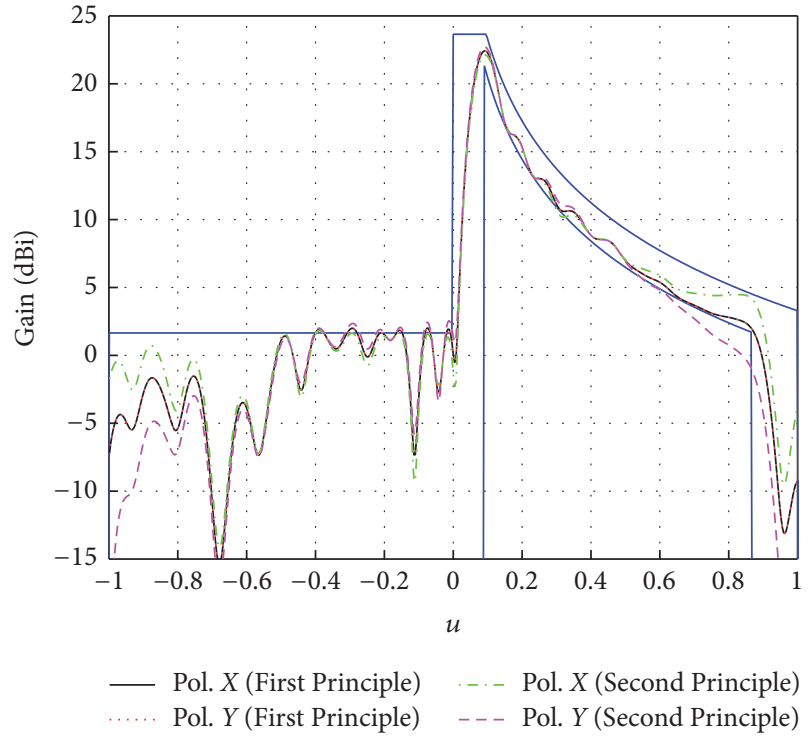

Figure 5: Differences between the First and the Second Principles of Equivalence for both linear polarizations in the elevation cut of a LMDS pattern synthesized using the POS formulation of the First Principle.

synthesized without taking the frame into account. Then, the dielectric frame was added to the optimized phases and the radiation patterns were computed again.

Figure 6 shows the results comparing the isoflux and LMDS radiation patterns with and without dielectric frame for the symmetric cut and $X$ polarization. Different frames were simulated having one, two, or three extra elements added at the edges of the reflectarray. As it is expected, the thicker the frame is, the more it affects the copolar patterns. In the case of the isoflux pattern, although the side lobes are affected, the increase in the ripple in the coverage area is more 


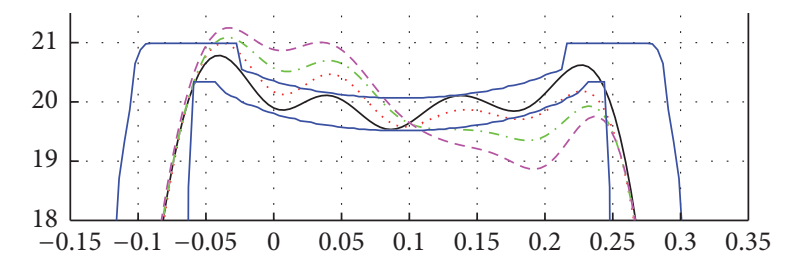

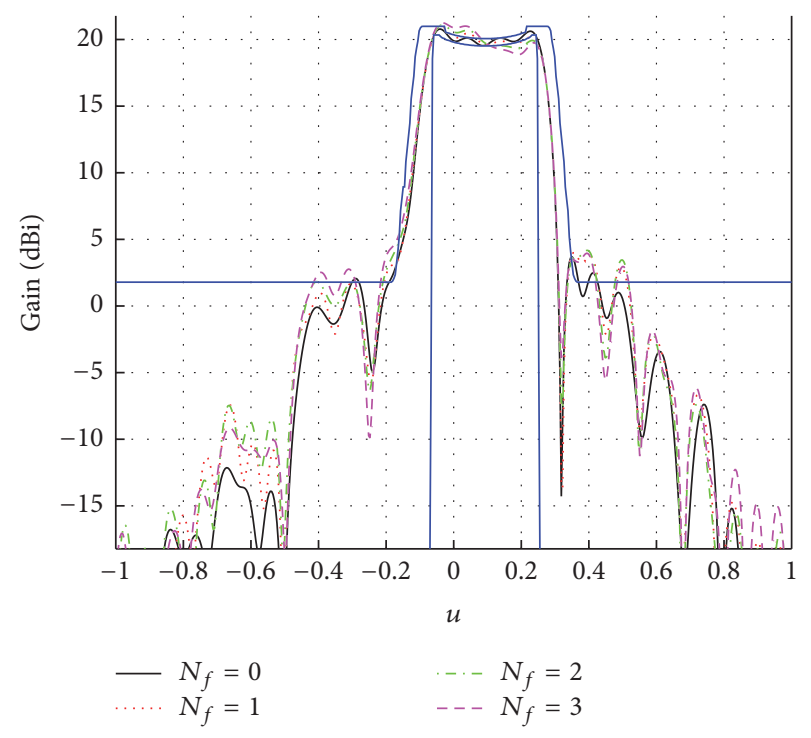

(a)

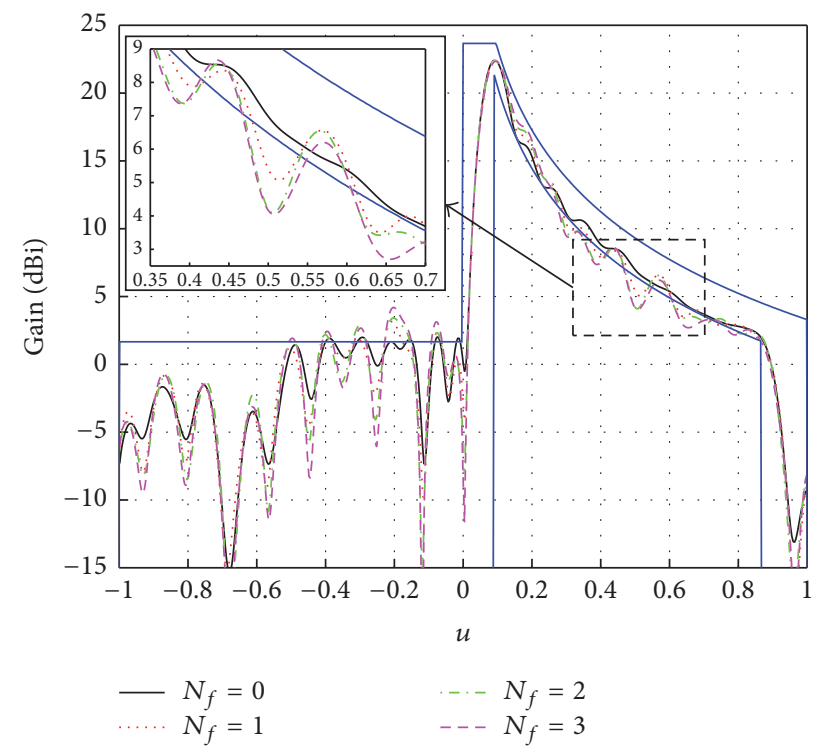

(b)

Figure 6: Dielectric frame effect on the shaped patterns for $X$ polarization when it is added after a synthesis was carried out. $N_{f}$ indicates the number of extra elements added as dielectric frame at each edge of the reflectarray. (a) Asymmetric cut of isoflux pattern. (b) Elevation cut of LMDS pattern.

relevant, which is more than $2 \mathrm{~dB}$ for the frame with three extra added elements (the allowed ripple was set to $0.55 \mathrm{~dB}$, which is a very tight requirement). The effects of the dielectric frame on the LMDS pattern in the elevation cut are similar to those of the isoflux pattern. The side lobes are increased around $2.5 \mathrm{~dB}$ and there appears a ripple of more than $2 \mathrm{~dB}$ in the coverage area for the frame with three extra added elements.

For both patterns, the results for $Y$ polarization are similar to those obtained for $X$ polarization.

The results displayed in Figure 6 show the necessity of including the effects of the dielectric frame during the synthesis so they can be compensated. This can be done by including the dielectric frame since the beginning of the synthesis. Another approach, which will be the one taken here, is to improve the previous synthesis without frame, that is, using its results as starting point for a new one, since the starting point for the optimization is very close to the desired solution. In any case, the phases of the dielectric frame must remain unaltered during the synthesis, and thus they will not be optimizing variables. The dielectric frame with $N_{f}=3$ extra added elements to the reflectarray sides has been chosen to carry out the new synthesis, since it represents the more extreme case. Figure 7 shows the optimized phase distribution for both radiation patterns (isoflux and LMDS) for $X$ polarization, including the dielectric frame, whose variation is masked by the total variation of the optimized phases. The phase distribution for $Y$ polarization is very similar to that obtained for $X$ polarization.

The results with the improved technique are shown in Figure 8 for $X$ polarization for the isoflux and LMDS patterns. There are also included the patterns without frame and with frame before the optimization. As it can be seen, the coverage area is improved in both cases when the dielectric frame is taken into account in the synthesis. In the LMDS pattern, the back lobes are also decreased and they also improve in the isoflux pattern, although side lobes at around $u=0.5$ remain high.

Finally, Figure 9 shows the cut in the plane of symmetry for the isoflux pattern and the cut in azimuth for the LMDS pattern for the same cases shown in Figure 8. As it can be seen, in the symmetric cut of both shaped patterns, the effect of the dielectric frame is almost negligible. In all cases, results for $Y$ polarization are very similar to those shown for $X$ polarization, and the same conclusions apply regarding the effects of the dielectric frame and its inclusion in the synthesis algorithm.

4.4. Experimental Validation. For the experimental validation, a reflectarray prototype was available that generates an isoflux pattern at $30 \mathrm{GHz}$. The synthesis had been carried out using the Second Principle of Equivalence and not taking into account the dielectric frame. The measurements did not match with the simulations using the analysis technique 


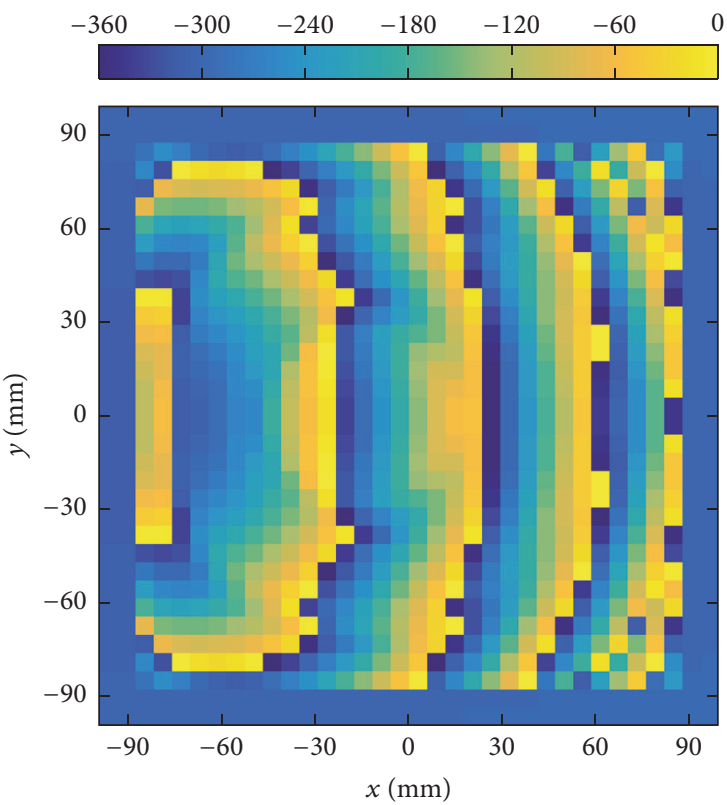

(a)

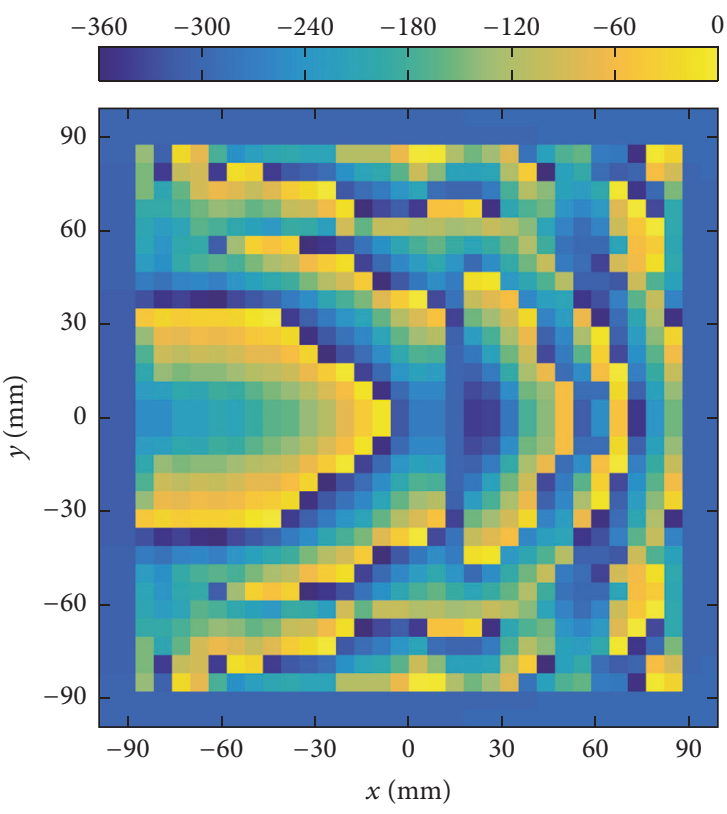

(b)

FIGURE 7: Synthesized phase distribution on the reflectarray surface including the dielectric frame with $N_{f}=3$, corresponding to the (a) isoflux and (b) LMDS patterns for $X$ polarization.
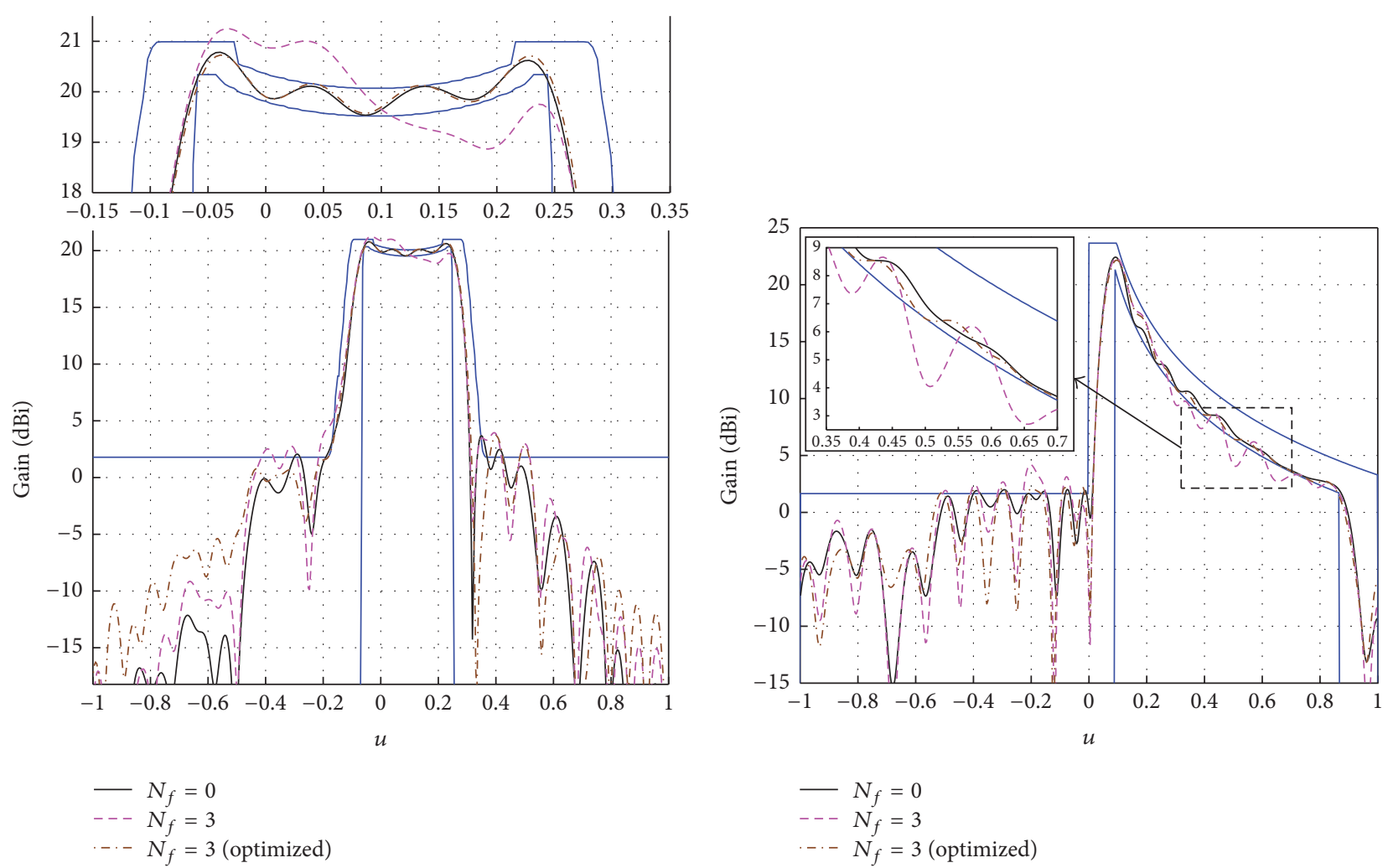

(a)

(b)

FIGURE 8: Comparison of the simulations between the reflectarray with frame taken into account in the synthesis and without frame, for $X$ polarization. $N_{f}$ indicates the number of extra elements added as dielectric frame at each edge of the reflectarray. (a) Asymmetric cut of isoflux pattern. (b) Elevation cut of LMDS pattern. 


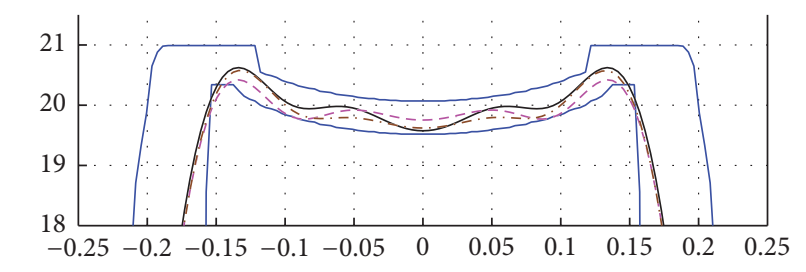

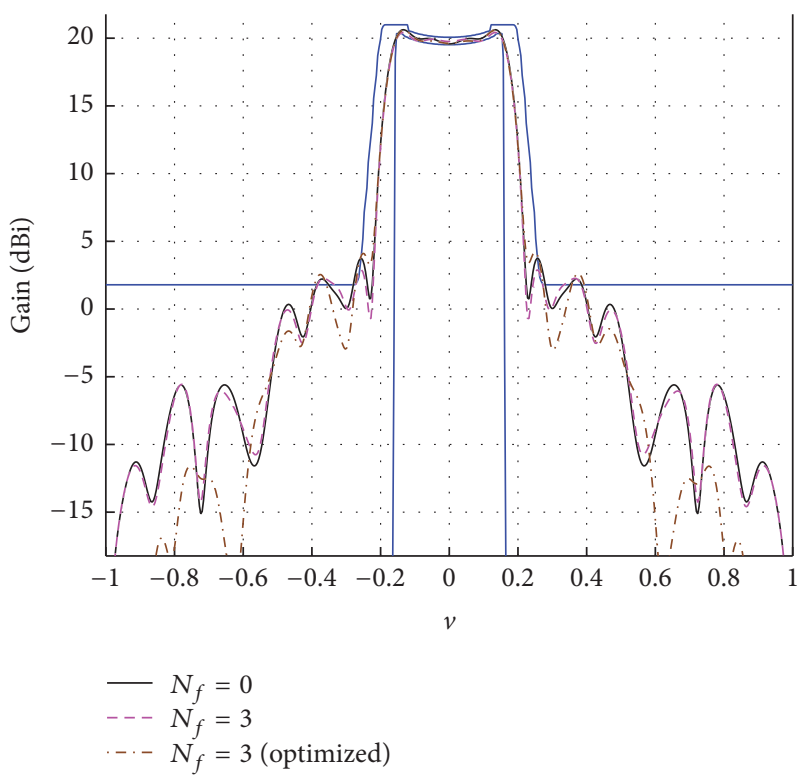

(a)

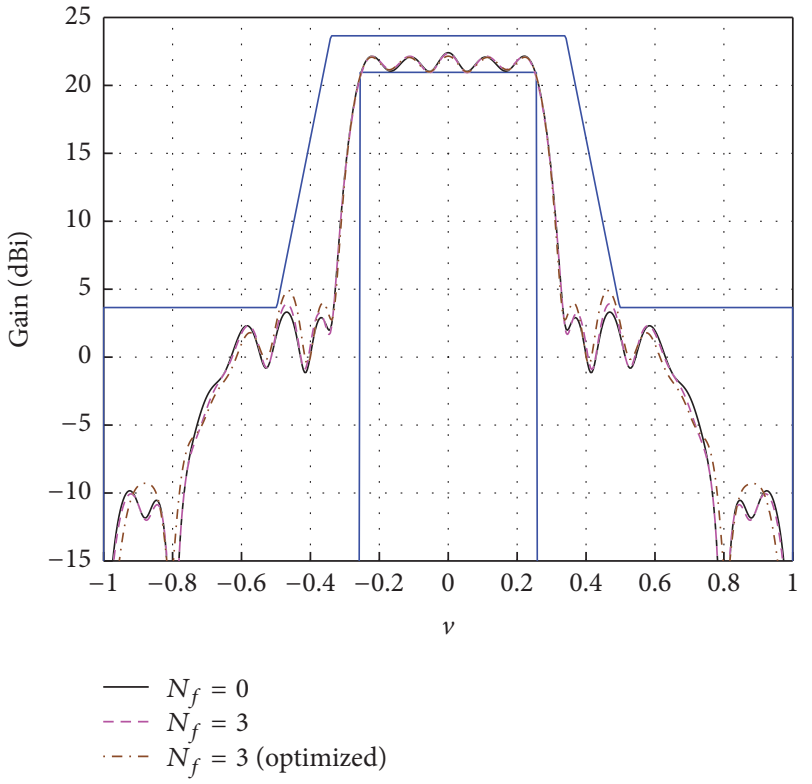

(b)

FIGURE 9: Comparison of the simulations between the reflectarray with frame taken into account in the synthesis and without frame, for $X$ polarization. $N_{f}$ indicates the number of extra elements added as dielectric frame at each edge of the reflectarray. (a) Symmetric cut of isoflux pattern. (b) Azimuth cut of LMDS pattern.

employed at that moment, and a large ripple appeared on the measured coverage zone which was not predicted. The aim is to apply the improved analysis technique adding the effects of the dielectric frame and First Principle of Equivalence.

The unit cell of the prototype is a single rectangular patch backed by a ground plane. The substrate is the Arlon $25 \mathrm{~N}$, with $\epsilon_{r}=3.38$, thickness $18 \mathrm{mil}$ and loss tangent 0.0025 at $10 \mathrm{GHz}$. The reflectarray is circular and comprised of 1528 elements arranged in a $4 \mathrm{~mm} \times 4 \mathrm{~mm}$ regular grid. However, it is printed on a rectangular substrate sheet resulting in a dielectric frame surrounding the reflectarray.

Figure 10 shows a picture of the prototype in the anechoic chamber facility at the University of Oviedo. Being superimposed, the phase distribution in the dielectric frame is shown which was simulated for $X$ polarization. In this case, the dielectric frame also includes the four corners.

Figure 11 shows the results comparing different simulations of the prototype and the measurement of the main cut along $u(v=0)$. As it can be seen, when the simulation takes into account the effects of the First Principle of Equivalence and dielectric frame, simulation and measurement agree better than when they are not taken into account. In fact, the coverage zone is seriously affected by both, as it can be seen by comparing the simulation with no frame with the Second Principle and the rest. If both effects would have been taken into account in the synthesis process, the prototype would have been better designed, presenting results similar to those obtained in previous sections. Finally, side lobes are also affected by diffraction at the edges of the breadboard and reflections on the supporting structure, which were not taken into account in the analysis.

\section{Conclusions}

This paper has presented an improved and practical reflectarray Phase-Only Synthesis (POS) based on the generalized Intersection Approach (IA) which employs the newly developed POS formulation of the First Principle of Equivalence and takes into account the dielectric frame which is usually present to screw the reflectarray breadboard to the supporting structure, providing more accurate results. The dielectric frame is modeled as extra reflectarray elements with no metallization added to the edges of the antenna. This improved analysis is used later to perform a POS.

For the synthesis, the chosen algorithm is the generalized IA, which already presents good convergence properties since it works with the squared field amplitude. However, it is computationally slow since it employs a general minimization algorithm in the backward projector. In order to speed up the algorithm, two strategies were presented. The first one consists in performing the synthesis in several steps, using less optimizing variables at the beginning. The benefit is 


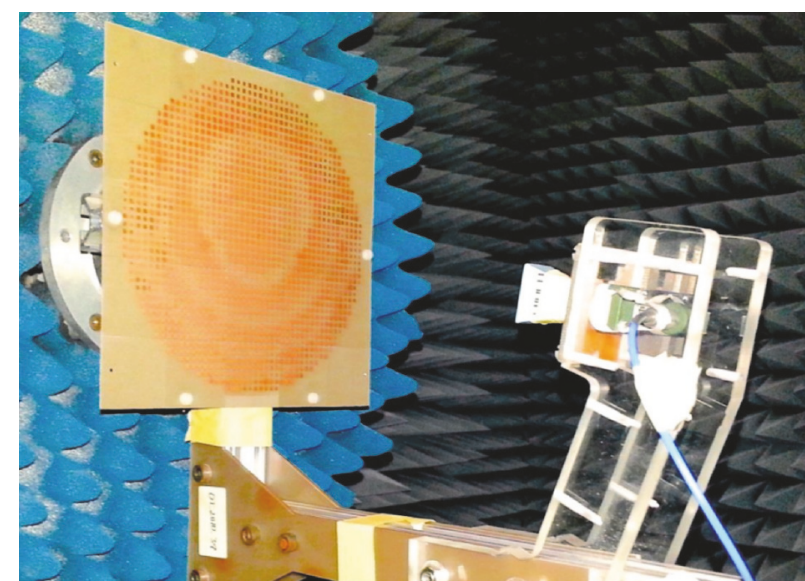

(a)

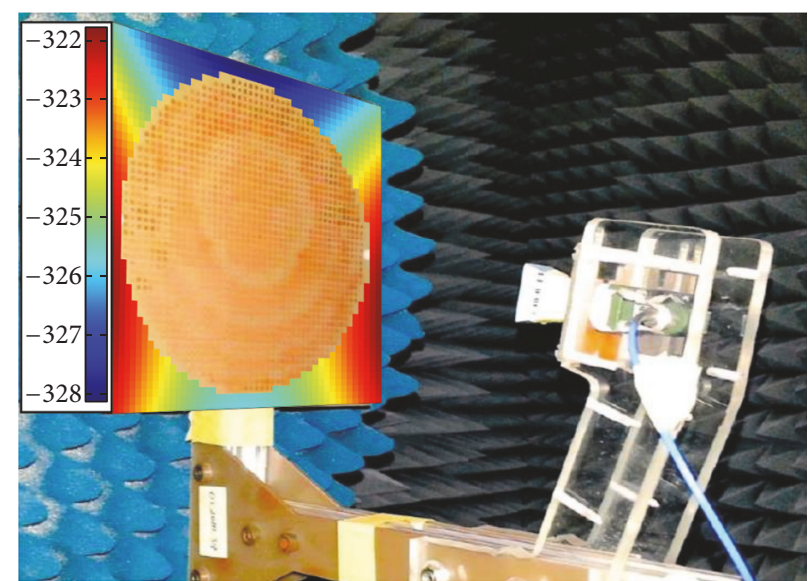

(b)

FIGURE 10: Picture of the reflectarray prototype which generates an isoflux pattern for global Earth coverage. (a) Original photograph. (b) In the picture it is shown as being superimposed on the frame the phase distribution (in degrees) simulated for the dielectric frame for $X$ polarization.

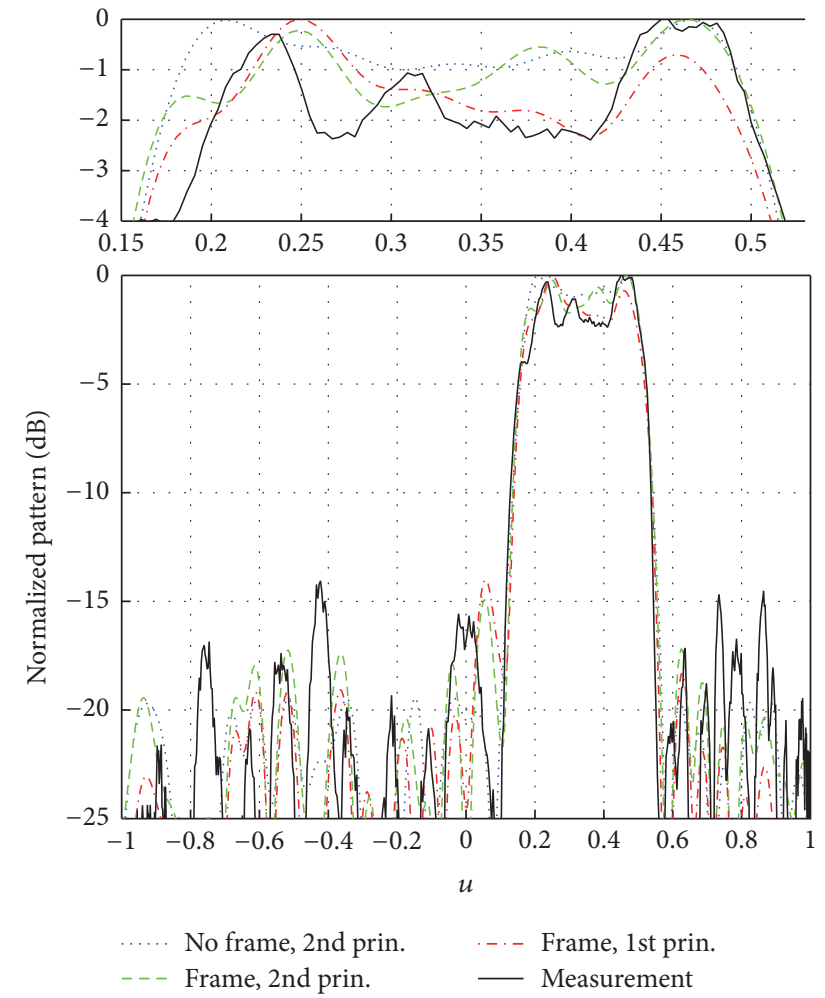

FIGURE 11: Comparison of the measurements and simulations in the main cut for $v=0$.

double: computing times and memory usage are decreased while convergence is improved since the number of local minima is reduced in the first steps of the synthesis. The second strategy consists in performing the synthesis with a reduced resolution of the far fields, considerably speeding up the algorithm. Once the synthesis has finished, the far fields may be computed with a higher resolution to plot the results.
In order to assess the improved analysis and synthesis, two shaped-beam patterns have been considered: an isoflux pattern for global Earth coverage and a Local Multipoint Distribution Service pattern for base stations. First, the differences between the First and Second Principles were demonstrated, showing the importance of carrying out the synthesis with the First Principle. Then, the effect of the dielectric frame was stated comparing the isoflux and LMDS patterns with and without frames of different sizes, and a synthesis taking into account the dielectric frame was carried out, which compensates the interferences of the frame in the copolar pattern. Finally, the improved analysis was validated experimentally with a prototype with an isoflux pattern, showing good agreement between simulations and measurements. The design of prototypes can be improved by employing the new analysis technique in the synthesis process, as proposed in this work.

\section{Conflicts of Interest}

The authors declare that there are no conflicts of interest regarding the publication of this paper.

\section{Acknowledgments}

This work was supported in part by the European Space Agency (ESA) under Contract ESTEC/AO/1-7064/12/NL/ $\mathrm{MH}$; by the Ministerio de Economía y Competitividad under Project TEC2014-54005-P (MIRIIEM); by the Gobierno del Principado de Asturias/FEDER under Project GRUPIN14114.

\section{References}

[1] J. Huang and J. A. Encinar, Reflectarray Antennas, John Wiley \& Sons, Hoboken, NJ, USA, 2008. 
[2] O. M. Bucci, G. Franceschetti, G. Mazzarella, and G. Panariello, "Intersection approach to array pattern synthesis," IEE Proceedings H - Microwaves, Antennas and Propagation, vol. 137, no. 6, pp. 349-357, 1990.

[3] J. A. Zornoza and J. A. Encinar, "Efficient phase-only synthesis of contoured-beam patterns for very large reflectarrays," International Journal of RF and Microwave Computer-Aided Engineering, vol. 14, no. 5, pp. 415-423, 2004.

[4] D. R. Prado, J. Álvarez, M. Arrebola, M. R. Pino, R. G. Ayestarán, and F. Las-Heras, "Efficient, accurate and scalable reflectarray phase-only synthesis based on the Levenberg-Marquardt algorithm," Applied Computational Electromagnetics Society Journal, vol. 30, no. 12, pp. 1246-1255, 2015.

[5] J. Perini and M. Idselis, "Note on antenna pattern synthesis using numerical iterative methods," IEEE Transactions on Antennas and Propagation, vol. 19, no. 2, pp. 284-286, 1971.

[6] T. S. Fong and R. A. Birgenheier, "Method of conjugate gradients for antenna pattern synthesis," Radio Science, vol. 6, no. 12, pp. 1123-1130, 1971.

[7] D. W. Boeringer and D. H. Werner, "Particle swarm optimization versus genetic algorithms for phased array synthesis," IEEE Transactions on Antennas and Propagation, vol. 52, no. 3, pp. 771-779, 2004.

[8] P. Nayeri, F. Yang, and A. Z. Elsherbeni, "Design of single-feed reflectarray antennas with asymmetric multiple beams using the particle swarm optimization method," IEEE Transactions on Antennas and Propagation, vol. 61, no. 9, pp. 4598-4605, 2013.

[9] J. A. Encinar, L. S. Datashvili, J. A. Zornoza et al., "Dualpolarization dual-coverage reflectarray for space applications," IEEE Transactions on Antennas and Propagation, vol. 54, no. 10, pp. 2827-2837, 2006.

[10] J. A. Encinar, M. Arrebola, L. F. De La Fuente, and G. Toso, "A transmit-receive reflectarray antenna for direct broadcast satellite applications," IEEE Transactions on Antennas and Propagation, vol. 59, no. 9, pp. 3255-3264, 2011.

[11] J. A. Encinar, R. Florencio, M. Arrebola et al., "Dualpolarization reflectarray in Ku-band based on two layers of dipole-arrays for a transmit-receive satellite antenna with south american coverage," in Proceedings of the 11th European Conference on Antennas and Propagation (EuCAP '17), pp. 80-83, Paris, France, 2017.

[12] O. M. Bucci, G. D’Elia, G. Mazzarella, and G. Panariello, "Antenna pattern synthesis: a new general approach," Proceedings of the IEEE, vol. 82, no. 3, pp. 358-371, 1994.

[13] A. Capozzoli, C. Curcio, A. Liseno, and G. Toso, "Fast, phaseonly synthesis of aperiodic reflectarrays using NUFFTs and CUDA," Progress in Electromagnetics Research, vol. 156, pp. 83103, 2016.

[14] D. R. Prado, M. Arrebola, M. R. Pino et al., "Efficient crosspolar optimization of shaped-beam dual-polarized reflectarrays using full-wave analysis for the antenna element characterization," IEEE Transactions on Antennas and Propagation, vol. 65, no. 2, pp. 623-635, 2017.

[15] R. Florencio, J. A. Encinar, R. R. Boix, V. Losada, and G. Toso, "Reflectarray antennas for dual polarization and broadband telecom satellite applications," IEEE Transactions on Antennas and Propagation, vol. 63, no. 4, part 1, pp. 1234-1246, 2015.

[16] W. L. Stutzman and G. A. Thiele, Antenna Theory and Design, John Wiley \& Sons, Hoboken, NJ, USA, 3rd edition, 2012.

[17] A. Ludwig, "The definition of cross polarization," IEEE Transactions on Antennas and Propagation, vol. 21, no. 1, pp. 116-119, 1973.
[18] C. A. Balanis, Ed., Modern Antenna Handbook, John Wiley \& Sons, Hoboken, NJ, USA, 2008.

[19] R. F. Díaz, Contribución al Análisis Eficiente y a la Mejora de Prestaciones de Antenas Reflectarray [Ph.D. thesis], Universidad Politécnica de Madrid, 2015.

[20] W. Hu, M. Arrebola, R. Cahill et al., "94 GHz dual-reflector antenna with reflectarray subreflector," IEEE Transactions on Antennas and Propagation, vol. 57, no. 10, pp. 3043-3050, 2009.

[21] D. R. Prado, M. Arrebola, M. R. Pino, and F. Las-Heras, "Complex reflection coefficient synthesis applied to dual-polarized reflectarrays with cross-polar requirements," IEEE Transactions on Antennas and Propagation, vol. 63, no. 9, pp. 3897-3907, 2015.

[22] "Techniques for the crosspolar optimization in reflectarray antennas," in XI Iberian Meeting on Computational Electromagnetics, pp. 45-48, Las Caldas, Asturias, Spain, 2016.

[23] D. Rodríguez Prado, A. Campa, M. Arrebola, M. R. Pino, J. A. Encinar, and F. Las-Heras, "Design, manufacture, and measurement of a low-cost reflectarray for global earth coverage," IEEE Antennas and Wireless Propagation Letters, vol. 15, pp. 1418-1421, 2016.

[24] M. Arrebola, J. A. Encinar, and M. Barba, "Multifed printed reflectarray with three simultaneous shaped beams for LMDS central station antenna," IEEE Transactions on Antennas and Propagation, vol. 56, no. 6, pp. 1518-1527, 2008. 


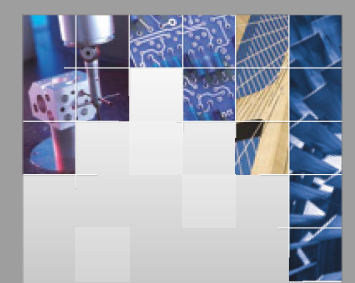

\section{Enfincering}
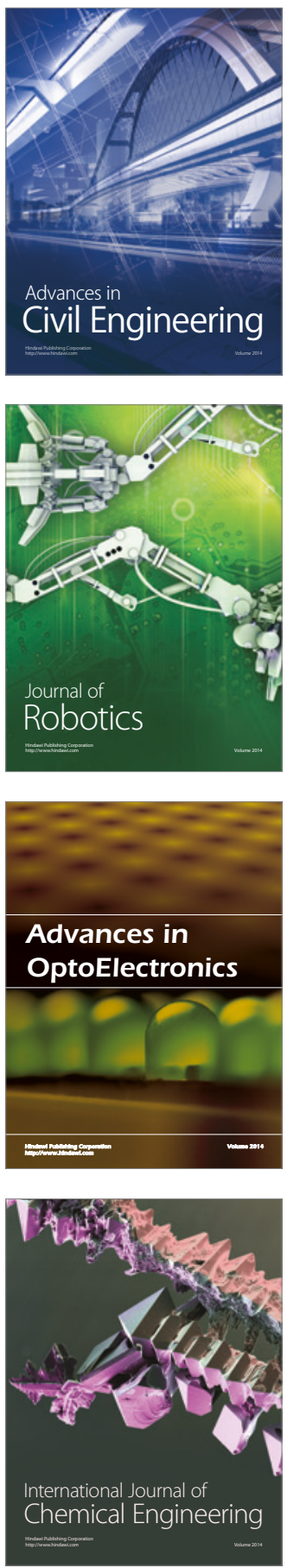

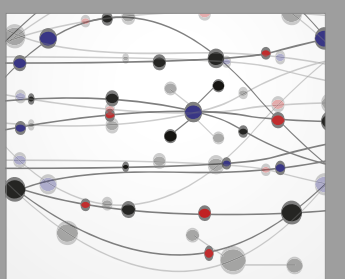

The Scientific World Journal

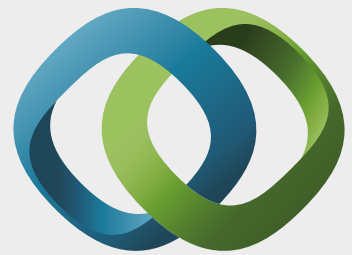

\section{Hindawi}

Submit your manuscripts at

https://www.hindawi.com
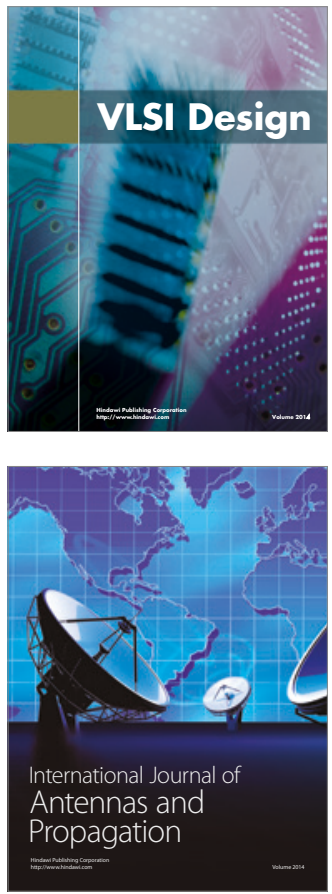

\section{Rotating}

Machinery
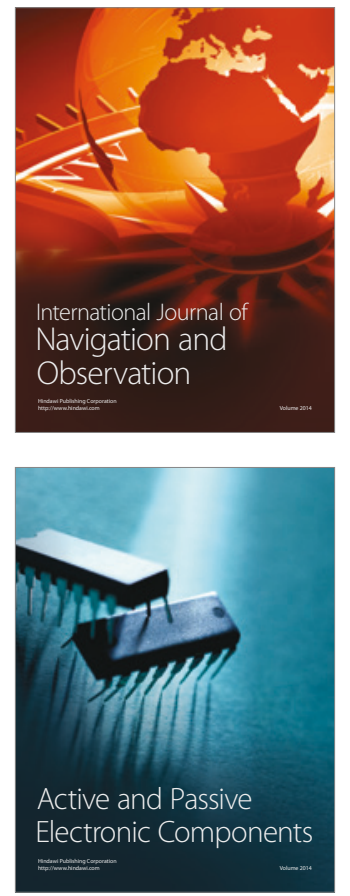
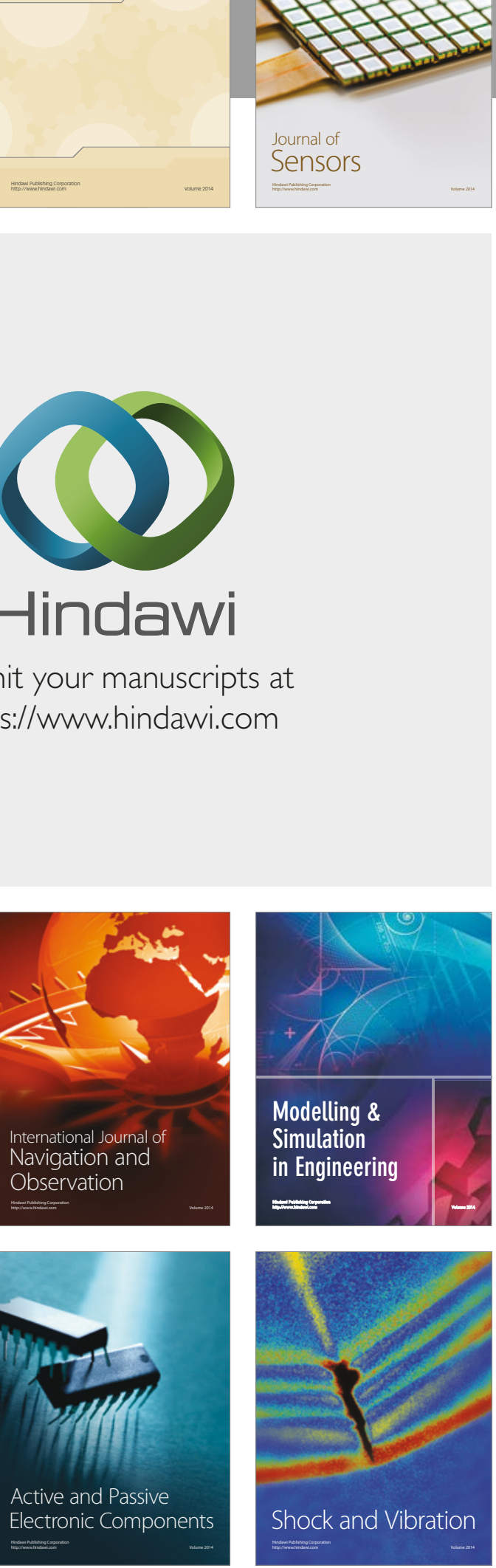
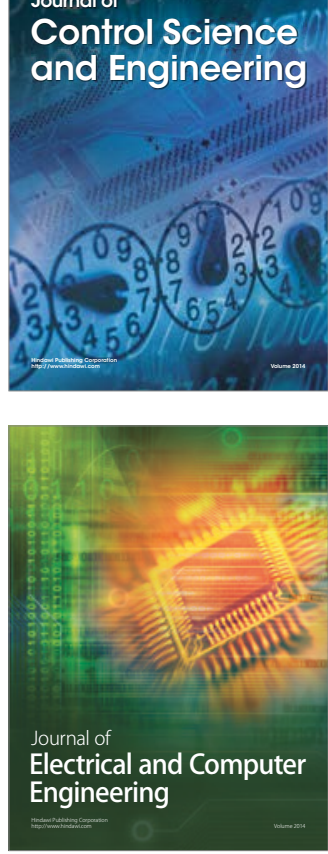

Distributed

Journal of

Control Science

and Engineering
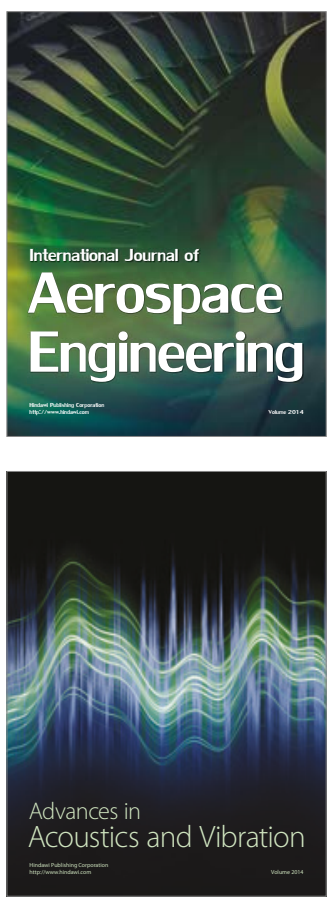

Sensor Networks 